\title{
Differentiable Generalized Inverses
}

\author{
John Z. Hearon* and John W. Evans*
}

(March 26, 1968)

\begin{abstract}
Necessary and sufficient conditions are given for a differentiable matrix to have a differentiable generalized inverse. It is shown that when these conditions are met there exist, for several classes of generalized inverses, a differentiable generalized inverse which coincides with a prescribed generalized inverse on a particular subset. The relations between the derivative of a matrix and that of a differentiable generalized inverse are given.
\end{abstract}

Key Words: Differentiable generalized inverse, generalized inverse, matrix.

\section{Introduction}

If $A$ is a nonsingular matrix the entries of which are differentiable functions of $t$, then the familiar relations between the derivative of $A$ and that of $A^{-1}$ are

$$
\begin{gathered}
\frac{d A}{d t}=-A \frac{d A^{-1}}{d t} A \\
\frac{d A^{-1}}{d t}=-A^{-1} \frac{d A}{d t} A^{-1} .
\end{gathered}
$$

If $A$ is a singular (either rectangular or square with zero determinant), differentiable matrix and $B$ is a differentiable generalized inverse of $A$, we inquire what relations analogous to (1) and (2) hold between the derivatives of $A$ and $B$.

In section 3 , necessary and sufficient conditions are given for the existence of a differentiable generalized inverse of a differentiable matrix. When these conditions obtain, we consider, in section 4 , the problem of the existence of a differentiable generalized inverse which coincides with a prescribed generalized inverse on some particular subset. Moreover, this problem is considered for several classes of generalized inverses.

In section 5 relations between the derivative of a matrix and that of a differentiable generalized inverse are given. These relations depend upon the class of generalized inverse and reduce, in the nonsingular case, to (1) and (2). Further, necessary and sufficient conditions are given such that (1) and (2) remain valid in the singular case when $A^{-1}$ is replaced by a differentiable generalized inverse. Finally, in section 6 , some special cases are considered.

\section{Preliminaries and Definitions}

All matrices are considered to have complex entries. For any matrix $M$, we denote by $\rho(M)$, $R(M), N(M)$ and $M^{*}$ the rank, range, null space and conjugate transpose, respectively, of $M$. If a function $f$ is such that its first $k$ derivatives exist and are continuous we write $f \epsilon \mathscr{C}^{k}$, and the interval on which this is true will be clear from the context. If the entries $m_{i j}$ of $M$ are such that

*An invited paper. Present address: Mathematical Research Branch, NIAMD, National Institutes of Health, Bethesda, Md. 20014. 
$m_{i j} \epsilon \mathscr{C}^{k}$, for all $i, j$, we write $M \epsilon \mathscr{C}^{k}$. If $f$ is a function defined on a subset $\mathscr{E}$ of an interval $[a, b]$, a function $g$ defined on $[a, b]$ is said to be an extension of $f$ to $[a, b]$ if $g(t)=f(t)$ whenever $t \epsilon \mathscr{E}$. We apply this definition to both scalar-values and matrix-values functions. ${ }^{1}$

We deal here with the same classes of generalized inverses previously defined [3] $]^{2}$ and employ the same definition of a strict generalized inverse of a given class as that given in [3].

\section{Existence of Differentiable Generalized Inverses}

We begin this section with the following lemma.

Lemma 1. A continuous projection defined on an interval has constant rank.

Proof. Let $A(t)$ be a continuous projection defined on an interval $\mathscr{E}$ of the real line, and let $\rho(A(t))=r(t)$. Then clearly the determinant det $(A+I)=2^{r(t)}$. The left-hand side of this equality is continuous. But a continuous integered-valued function on a connected set is constant. It follows that $\rho(A(t))$ is constant.

If $A$ is a given matrix, it is known [2] that there exist matrices $B \epsilon C_{1}(A)$ which commute with $A$ if and only if $\rho(A)=\rho\left(A^{2}\right)$ and that when this condition is met there exists a unique $C_{2}$-inverse which commutes with $A$; it is in fact a polynomial in $A$. The next lemma concerns the differentiability of this generalized inverse.

Lemma 2. Let $\mathrm{A} \epsilon^{\mathscr{C}^{\mathrm{k}}}$ on an interval $\mathscr{E}$ and have the property that $\rho(\mathrm{A}(\mathrm{t}))=\rho\left(\mathrm{A}^{2}(\mathrm{t})\right)=\mathrm{r}$ for each $\mathrm{t} \epsilon \mathscr{\mathscr { E }}$. If $\mathrm{B}(\mathrm{t})$, for each $\mathrm{t} \epsilon \mathscr{E}$, is the unique $\mathrm{C}_{2}$-inverse of $\mathrm{A}(\mathrm{t})$ which commutes with $\mathrm{A}(\mathrm{t})$, then $\mathrm{B} \epsilon \mathscr{C}^{\mathrm{k}}$ on $\mathscr{E}$.

Proof. From $\rho(A)=\rho\left(A^{2}\right)$ we have that the Jordan canonical form of $A(t)$ is $\operatorname{diag}\left(A_{1}(t), 0\right)$, where $A_{1}(t)$ is an $r$-square nonsingular matrix. The characteristic polynomial of $A(t)$ has coefficients in $\mathscr{C}^{k}$ and is of the form $\lambda^{n-r} f(\lambda)$, where $f(\lambda)$ is the characteristic polynomial of $A_{1}(t)$. From $f(\lambda)$ we can construct a polynomial $p(\lambda)$, with coefficients in $\mathscr{C}^{\mathrm{k}}$, such that $A^{-1}(t)=p\left(A_{1}(t)\right)$. Now the matrix $B_{1}(t)=p(A(t))$ is, by a known theorem [1], a $C_{1}$-inverse of $A(t)$ since the Jordan form of $A B_{1}$ is the projection diag $\left(I_{r}, 0\right)$. The matrix $B=B_{1} A B_{1}$ is, by Theorem/lof [3] a $C_{2}$-inverse of $A$, commutes with $A$, and clearly $B \epsilon \mathscr{C}^{k}$.

We now employ Lemmas 1 and 2 to prove the main result of this section.

Theorem 1. Let $\mathrm{A} \epsilon_{\mathscr{C}^{\mathrm{k}}}$ on an interval $\mathscr{E}$. If $\rho(\mathrm{A})$ is constant on $\mathscr{E}$, then the $\mathrm{C}_{4}$-inverse of $\mathrm{A}$ is in $\mathscr{C}^{\mathrm{k}}$ on $\mathscr{E}$. If $\rho(\mathrm{A})$ is not constant on $\mathscr{E}$, then no $\mathrm{C}_{1}$-inverse, $1 \leqslant \mathrm{i} \leqslant 4$, can be continuous on $\mathscr{E}$.

Proof. If $\rho(A)$ is constant on $\mathscr{E}$, then the Hermitian matrix $H=A^{*} A$ meets the conditions of Lemma 2. We are thus assured of a matrix $W(t)$ which is the unique $C_{2}$-inverse of $H(t)$ which commutes with $H(t)$, and $W \epsilon \mathscr{C}^{k}$ on $\mathscr{E}$. Moreover, $W$ is Hermitian [2]. But $W H=H W$ and $W \epsilon C_{2}(H)$ imply $W \epsilon C_{4}(H)$. By Theorem 1 of [3], $B=W A^{*}$ is the $C_{4}$-inverse of $A$ and clearly $B \epsilon \mathscr{C}^{k}$. Now let $B$ be any $C_{i}$-inverse of $A, 1 \leqslant i \leqslant 4$. If $B$ is continuous on $\mathscr{E}$ then, by Lemma 1 , the continuous projection $A B$ has constant rank on $\mathscr{E}$. But by a known theorem [6], $\rho(A B)=\rho(A)$, and $A$ has constant rank.

\section{Extension of a Given Generalized Inverse to a Differentiable Generalized Inverse}

Suppose that $A \epsilon^{k} \mathscr{C}^{k}$ on $[a, b], \rho(A)$ is constant on $[a, b]$ and that for some subset $\mathscr{E}$ of $[a, b]$ there is defined a matrix $\hat{B}_{i}$ which is in $C_{i}(A)$ on $\mathscr{E}$ for some $1 \leqslant i \leqslant 4$. If there is an extension of $\hat{B}_{i}$ to all of $[a, b]$ which is in $\mathscr{C}^{k}$ and which is a $C_{i}$-inverse of $A$ on $[a, b]$ then evidently each entry of $\hat{B}_{i}$ has an extension to all of $[a, b]$ which is in $\mathscr{C}^{k}$. The converse of this statement is the content of the next theorem.

THEOREM 2. Let $\mathrm{A}, \widehat{\mathrm{B}}_{\mathrm{i}}, \mathscr{E}$ and $[\mathrm{a}, \mathrm{b}]$ be as above. If every entry of $\hat{\mathrm{B}}_{\mathrm{i}}$ has some extension to all of $[\mathrm{a}, \mathrm{b}]$ which is in $\mathscr{C}^{\mathrm{k}}$, then there exists a $\mathrm{B}_{\mathrm{i}}$ which is an extension of $\hat{\mathrm{B}}_{\mathrm{i}}$ to $[\mathrm{a}, \mathrm{b}]$ and such that $\mathrm{B}_{\mathrm{i}} \epsilon \mathscr{C}^{\mathrm{k}}$ and $\mathrm{B}_{\mathrm{i}} \epsilon \mathrm{C}_{\mathrm{i}}(\mathrm{A})$ on $[\mathrm{a}, \mathrm{b}]$.

Proof. For the case $i=4$ we have no need for the theorem. For, the $C_{4}$-inverse is unique and is, by Theorem 1 , in $\mathscr{C}^{k}$.

\footnotetext{
'In what follows we deal with closed intervals. The proofs given go through, with minor modification, for intervals which are half-open, open, semi-infinite.

${ }^{2}$ Figures in brackets indicate the literature references at the end of this paper.
} 
Let $i=1$. By hypothesis there is an extension $\widetilde{B}_{1}$ of $\hat{B}_{1}$ and $\widetilde{B}_{1} \in \mathscr{C}^{k}$. Let $B^{+}$be the $C_{4}$-inverse of $A$, and let $P_{R}=A B^{+}, P_{N}=I-B^{+} A$. Then $P_{R}$ and $P_{N}$ are in $\mathscr{C}^{\mathrm{k}}$ and are projections onto $R(A)$ and $N(A)$, respectively. If $D=\widetilde{B}_{1}-B^{+}$, then $D$ is, on $\mathscr{E}$, the difference between two matrices in $C_{1}(A)$. Then $B_{1}=B^{+}+P_{N} D P_{R}+D\left(I-P_{R}\right)$ is, by Theorem 2 of [3], a $C_{1}$-inverse on all of $[a, b]$ such that $B_{1}=\hat{B}_{1}$ on $\mathscr{E}$. Clearly $B_{1} \in \mathscr{C}^{\mathrm{k}}$.

Let $i=2$. By what we have proved so far, we may take $\widetilde{B}_{2}$ as an extension of $\hat{B}_{2}$ such that $\widetilde{B}_{2} \epsilon C_{1}(A)$ and $\widetilde{B}_{2} \epsilon \mathscr{C}^{k}$ on $[a, b]$. Then, by Theorem 2 of $[3], B_{2}=\widetilde{B}_{2} A \widetilde{B}_{2}$ is a $C_{2}$-inverse of $A$ on $[a, b]$. Clearly $B_{2}=\hat{B}_{2}$ on $\mathscr{E}$ and $B_{2} \epsilon_{\mathscr{C}^{k}}$ on $[a, b]$.

Let $i=3$. By the first part of this proof we may take $\widetilde{B}_{3}$ as an extension of $\hat{B}_{3}$ such that $\widetilde{B}_{3} \in C_{1}(A)$ and $\widetilde{B}_{3} \in \mathscr{C}^{k}$ on $[a, b]$. Then, by Theorem 2 of $[3], B_{3}=\widetilde{B}_{3} A B^{+}$, where $B^{+} \epsilon C_{4}(A)$, is a $C_{3}$-inverse of $A$ on $[a, b]$ and $B_{3}=\hat{B}_{3}$ on $\mathscr{E}$. Evidently $B_{3} \in \mathscr{C}^{k}$ on $[a, b]$.

The proof for $i=3^{\prime}$ is an obvious parallel of that for $i=3$.

If $A$ is a differentiable $E P r$ matrix we may inquire as to existence of a differentiable $E P r$ $C_{i}$-inverse of $A$, where of necessity $i \geqslant 2$, for by a known theorem if $B \epsilon C_{1}(A)$ then $B \epsilon C_{2}(A)$ if and only if $\rho(B)=\rho(A)[1,6]$. This question is settled by Corollary 1 .

Corollary 1. Let $\mathrm{A} \epsilon \mathscr{C}^{\mathrm{k}}$ be an EPr matrix on $[\mathrm{a}, \mathrm{b}]$ and let $\hat{\mathrm{B}}$ be an $\mathrm{EPr} \mathrm{C}_{2}$-inverse of $\mathrm{A}$ on a subset $\mathscr{E}$ of $[\mathrm{a}, \mathrm{b}]$. If every entry of $\hat{\mathrm{B}}$ has an extension to $[\mathrm{a}, \mathrm{b}]$ which is in $\mathscr{C}^{\mathrm{k}}$, then there exists $a \mathrm{~B}$ which is an extension of $\mathrm{B}$ to $[\mathrm{a}, \mathrm{b}]$ and such that, on $[\mathrm{a}, \mathrm{b}], \mathrm{B} \epsilon \mathscr{C}^{\mathrm{k}}, \mathrm{B} \epsilon \mathrm{C}_{2}(\mathrm{~A})$ and $\mathrm{B}$ is EPr.

Proof. Let $\widetilde{B} \epsilon C_{2}(A)$ be the extension of $\hat{B}$ given by Theorem 2. Then by Theorem 3 of [3], $B=\widetilde{B} A^{*} \widetilde{B}^{*}$ is a $C_{2}$-inverse of $A$ and is $\operatorname{EPr}$ on $[a, b]$. By the same theorem, $B=\hat{B}$ on $\mathscr{E}$. By construction, $B$ is clearly in $\mathscr{C}^{k}$.

It is an easy result from Theorem 1 that if on $[a, b] A$ is a differentiable $\operatorname{EPr}$ matrix, then the $C_{4}$-inverse $B$ of $A$ is a differentiable $E P r$ matrix. For, Theorem 1 shows that $B \epsilon \mathscr{C}^{k}$ whenever $A \epsilon \mathscr{C}^{k}$ and it follows from a known theorem [5] that $B$ is $E \operatorname{Pr}$. Further, if $A$ is $E \operatorname{Pr}$, then $\rho(A)=\rho\left(A^{2}\right)$ $=r$ [4], and if $A \epsilon^{C^{k}}$, $A$ meets the conditions of Lemma 2. But by Lemma 6 of [3], the matrix vouched for by Lemma 2 is the $C_{4}$-inverse of $A$. Thus we can so far assert that, trivially, a differentiable $E P r$ matrix has a differentiable $E P r$ generalized inverse of every class, for it has a differentiable $E P r C_{4}$-inverse. The question of the existence of differentiable matrices which are at some points on $[a, b]$ strict $E \operatorname{Pr} C_{2}$-inverses of a differentiable $E \operatorname{Pr}$ matrix is cared for by Theorem 7 of [3], which gives properties of the map $\psi(B)=B A^{*} B^{*}$ (where $A$ is $E P r$ and $B \epsilon C_{2}(A)$ ) upon which Corollary 1 is based. ${ }^{3}$

\section{The Derivative of a Generalized Inverse}

In this section we denote by $M^{\prime}$ the derivative of a differentiable matrix $M$. We assume the existence of a differentiable generalized inverse, $B$, of a differentiable matrix $A$ and study the relations which obtain between $A^{\prime}$ and $B^{\prime}$. If $B$ is any generalized inverse of $A$ we define, throughout this section, the projections

$$
\begin{aligned}
& E_{1}=A B \\
& E_{2}=B A .
\end{aligned}
$$

Then $A B A=E_{1} A=A E_{2}=A$ shows that $E_{1}\left(E_{2}\right)$ is a left (right) identity element for $A$. If $B \epsilon C_{2}(A)$, then $B A B=B=E_{2} B=B E_{1}$ shows that $E_{2}\left(E_{1}\right)$ is a left (right) identity element for $B$. Relations (1) and (2) stem from the fact that the derivative of $A A^{-1}=A^{-1} A=I$ is zero. In a somewhat analogous manner the relations derived in what follows stem from Lemma 3.

LEMMA 3. If $\mathrm{E}$ is a differentiable projection, then $\mathrm{EE}^{\prime} \mathrm{E}=0$.

Proof. From $E=E^{2}$ we have $E^{\prime}=E^{\prime} E+E E^{\prime}$. Left multiplication of this last equality by $E$ gives $E E^{\prime}=E E^{\prime} E+E E^{\prime}$, from which the result follows.

\footnotetext{
${ }^{3}$ We observe that by the last display of the proof of Theorem 5 of [3] and by Theorem 7 of [3], the set $C_{2}(A)-C_{3^{\prime}}(A)$ is empty if and only if $A$ is nonsingular
} 
The next theorem furnishes an analog of (1) for a differentiable $C_{1}$-inverse and an analog of (2) for a differentiable $C_{2}$-inverse.

THEOREM 3. If $\mathrm{A}$ is differentiable and $\mathrm{B}$ is any differentiable $\mathrm{C}_{1}$-inverse of $\mathrm{A}$ then

$$
\mathrm{E}_{1} \mathrm{~A}^{\prime} \mathrm{E}_{2}=-\mathrm{AB}^{\prime} \mathrm{A}
$$

and if $\mathrm{B}$ is a differentiable $\mathrm{C}_{2}$-inverse then also

$$
\mathrm{E}_{2} \mathrm{~B}^{\prime} \mathrm{E}_{1}=-\mathrm{BA}^{\prime} \mathrm{B} .
$$

Proof. If $B \epsilon C_{1}(A)$, then $E_{1} A=A$. By Lemma 3 we have that $E_{1} E_{1}^{\prime} A=E_{1} E_{1}^{\prime} E_{1} A=0$. This being so, we have, using (3) and (4) $E_{1} E_{1}^{\prime} A=E_{1}\left(A^{\prime} B+A B^{\prime}\right) A=E_{1} A^{\prime} E_{2}+A B^{\prime} A=0$, from which (5) follows. If $B \epsilon C_{2}(A)$, then multiplication of (5) from left and right by $B$ and using $E_{2} B=B E_{1}=B$ gives (6).

We observe that (2) can always be obtained from (1) and conversely. But, as seen from the proof of Theorem 3, (6) follows from (5) only if $B \epsilon C_{2}(A)$, i.e., only if $B \epsilon C_{1}(A)$ and $A \epsilon C_{1}(B)$.

The next theorem gives conditions under which a differentiable $C_{1}$-inverse of $A$ can be substituted for $A^{-1}$ in (1) and conditions under which a differentiable $C_{2}$-inverse of $A$ can be substituted for $A^{-1}$ in (2). An example is given in the next section.

THEOREM 4. If $\mathrm{A}$ is differentiable and $\mathrm{B}$ is a differentiable $\mathrm{C}_{1}$-inverse of $\mathrm{A}$, then $\mathrm{A}^{\prime}=-\mathrm{AB}^{\prime} \mathrm{A}$ if and only if $\mathrm{E}_{1}^{\prime} \mathrm{A}=\mathrm{AE}_{2}^{\prime}=0$. If $\mathrm{B}$ is a differentiable $\mathrm{C}_{2}$-inverse of $\mathrm{A}$, then $\mathrm{B}^{\prime}=-\mathrm{BA}^{\prime} \mathrm{B}$ if and only if $\mathrm{E}_{2}^{\prime} \mathrm{B}=\mathrm{BE}_{1}^{\prime}=0$.

Proof. If $E_{1}^{\prime} A=A E_{2}^{\prime}=0$, then from $A=E_{1} A=A E_{2}$ we have $A^{\prime}=E_{1} A^{\prime}=A^{\prime} E_{2}$. But then (5) reads $A^{\prime}=-A B^{\prime} A$. Conversely, if $A^{\prime}=-A B^{\prime} A$, left multiplication by $E_{1}$ shows $E_{1} A^{\prime}=A^{\prime}$ and right multiplication by $E_{2}$ shows $A^{\prime} E_{2}=A^{\prime}$. It now follows from $A^{\prime}=E_{1}^{\prime} A+E_{1} A^{\prime}=A E_{2}^{\prime}+A^{\prime} E_{2}$ that $E_{1}^{\prime} A=A E_{2}^{\prime}=0$. Similarly, if $B$ is a $C_{2}$-inverse of $A$, then $B=E_{2} B=B E_{1}$ and $E_{2}^{\prime} B=B E_{1}^{\prime}=0$ together imply $B^{\prime}=E_{2} B^{\prime}=B^{\prime} E_{1}$ and (6) becomes $B^{\prime}=-B A^{\prime} B$. Conversely $B^{\prime}=-B A^{\prime} B$ implies $B^{\prime}=E_{2} B^{\prime}$ $=B^{\prime} E_{1}$ which implies $E_{2} B^{\prime}=B E_{1}^{\prime}=0$.

If $A$ is $m \times n$, then $E_{1}$ and $E_{2}$ are $m$-square and $n$-square respectively. In the event that $\rho(A)=m$ we have $E_{1}=I_{m}$ and Theorem 4 becomes $A^{\prime}=-A B^{\prime} A$ if and only if $A E_{2}^{\prime}=0$ when $B \epsilon C_{1}(A)$ and $B^{\prime}=-B A^{\prime} B$ if and only if $E_{2}^{\prime} B=0$ when $B \epsilon C_{2}(A)$. Similar statements hold when $\rho(A)=n$. The nonsingular case $\left(\rho(A)=m=n, E_{1}^{\prime}=E_{2}^{\prime}=0\right)$ is clearly a special case of Theorem 4 .

\section{Special Cases}

Let $A$ be a matrix such that $P^{-1} A P=\operatorname{diag}\left(J(t), J_{0}\right)$ where $P$ is a constant matrix, $J(t)$ is the Jordan block corresponding to the nonzero roots of $A, J_{0}$ is the Jordan block corresponding to the zero roots of $A$. Assume that the structure of the Jordan form remains the same over some $t$-interval over which $A$ is differentiable. It is easily verified that if $P^{-1} B P=\operatorname{diag}\left(J^{-1}(t), J_{0}^{T}\right)$, where $J_{0}^{T}$ is the transpose of $J_{0}$, then $B$ is a $C_{2}$-inverse of $A$. For, $J_{0}$ is a direct sum of nilpotent blocks, each block having l's on the subdiagonal and zeroes elsewhere. For any such block $N$ of order $k, N^{T} N=\operatorname{diag}$ $\left(I_{k-1}, 0\right)$ which is idempotent and has the same rank as $N$. By a known theorem [1], $N^{T}$ is then a $C_{2^{-}}$ inverse of $N$ and hence $J_{0}^{T}$ is a $C_{2}$-inverse of $J_{0}$. Now $B A$ is similar to $\operatorname{diag}\left(I, J_{0}^{T} J\right)$ and by the same theorem [1], $B$ is a $C_{2}$-inverse of $A$. In this case then, $E_{1}=P \operatorname{diag}\left(I, J_{0} J_{0}^{T}\right) P^{-1}$ and $E_{2}=P \operatorname{diag}$ $\left(I, J_{0}^{T} J_{0}\right) P^{-1}$ are constant matrices and Theorem 4 applies.

If $B$ is a $C_{2}$-inverse of $A$ and commutes with $A$, then $E_{1}=E_{2}$. Further, since $A, B, E_{1}$ and $E_{2}$ all have the same rank $[1,6]$, it follows that $A$ and $B$ have the same null space and that $A^{*}$ and $B^{*}$ have the same null space. Given this, the two conditions $E_{1}^{\prime} A=A E_{2}^{\prime}=0$ and $E_{2}^{\prime} B=B E_{1}^{\prime}=0$ are equivalent. For, the first requires that the columns of $E^{\prime}=E_{1}^{\prime}=E_{2}^{\prime}$ be in $N(A)$ and that the columns of $\left(E^{\prime}\right)^{*}$ be in $N\left(A^{*}\right)$; while the second requires that the columns of $E^{\prime}$ be in $N(B)$ and that the columns of $\left(E^{\prime}\right)^{*}$ be in $N\left(B^{*}\right)$. 
Thus if $B \epsilon C_{2}(A)$, is differentiable, and commutes with $A$, we have from Theorem 4 that if either of the conditions $A^{\prime}=-A B^{\prime} A$ and $B^{\prime}=-B A^{\prime} B$ is satisfied the other is also satisfied.

We have shown in section 4 the existence of a differentiable $E \operatorname{Pr} C_{2}$-inverse of the differentiable $E P r$ matrix. It is known [1] that an arbitrary square matrix possesses a $C_{2}$-inverse which is $E P r$. The next example shows a matrix which can have no continuous real $C_{2}$-inverse which is $E P r$. Let $A$ be

$$
A=\left[\begin{array}{ll}
\cos t & \sin t \\
0 & 0
\end{array}\right]
$$

on the interval $\mathscr{E}=[0, \pi]$. Then $\rho(A)=1$ on $\mathscr{E}$ and clearly $A$ is differentiable there. Let $B \epsilon C_{2}(A)$ and $N(B)=N\left(B^{*}\right)$. We now show that $B$ is not continuous. Since $[6] \rho(B A)=\rho(A)=1$, on $\mathscr{E}$, we have that $B A \neq 0$, for $t \epsilon \mathscr{E}$ and that the first column, $b_{1}$, of $B$ is not zero for $t \in \mathscr{E}$. But $B A \neq 0$ implies $0=R(A) \cdot N(B)=R(A) \cdot N\left(B^{*}\right)=R(A) \cdot R(B)^{\perp}$. Then since the vector $x$, where $x^{*}=[1,0]$, is a basis for $R(A), R(A) \cdot R(B)^{\perp}=0$ implies that if $y^{*}=[0,1]$, then $y \notin R(B)$. Since $b_{1} \neq 0$, for $t \epsilon \mathscr{E}$, is a basis for $R(B)$ we now have that $b_{11}$, the first entry of $b_{1}$, is never zero for $t \in \mathscr{E}$. We also have $\rho(A B)=\rho(B)=1$, on $\mathscr{E}$, so that $C=A B \neq 0$, for $t \in \mathscr{E}$, and in particular $c_{11}(t)=b_{11}(t) \cos t+b_{21}(t)$ $\sin t \neq 0$, for $t \in \mathscr{E}$. Now assume that $b_{11}$ is continuous on $\mathscr{E}$. Then $c_{11}(0)$ and $c_{11}(\pi)$ have opposite signs, since $b_{11}$ cannot change signs on $\mathscr{E}$. But then $c_{11}(t)=0$ for some $t \in \mathscr{E}$, which is a contradiction.

\section{References}

[1] J. Z. Hearon, Construction of $E P r$ generalized inverses by inversion of nonsingular matrices. J. Res. NBS $71 B$ (Math. and Math. Phys.) Nos. 2 and 3, 57-60 (1967).

[2] J. Z. Hearon, A generalized matrix version of Rennie's inequality, J. Res. NBS 71 B (Math. and Math. Phys.) Nos. 2 and 3 , 61-64 (1967).

[3] J. Z. Hearon and J. W. Evans, On spaces and maps of generalized inverses, J. Res. NBS 72B (Math. Sci.) No. 2, (1968).

[4] I. J. Katz and M. H. Pearl, On EPr and normal EPr matrices, J. Res. NBS 70B (Math. and Math. Phys.) No. 1, 47-77 (1966).

[5] M. H. Pearl, On generalized inverses of matrices, Proc. Cambridge Philos. Soc. 62, 673-677 (1966).

[6] C. A. Rohde, Some results on generalized inverses, SIAM Rev. 8, 201-205 (1966).

(Paper 72B2-264) 